\title{
Neoadjuvant Androgen Deprivation Therapy Effects on Perioperative Outcomes Prior to Radical Prostatectomy: Eleven Years of Experiences at Ramathibodi Hospital
}

\author{
Premsant Sangkum ' \\ Kun Sirisopana' \\ Pocharapong Jenjitranant (D) \\ Kittinut Kijvikai' \\ Suthep Pacharatakul ${ }^{2}$ \\ Charoen Leenanupunth' \\ Wachira Kochakarn' \\ Wisoot Kongchareonsombat (D) \\ 'Division of Urology, Department of \\ Surgery, Faculty of Medicine, Ramathibodi \\ Hospital, Mahidol University, Bangkok, \\ Thailand; ${ }^{2}$ Division of Urology, \\ Department of Surgery, Police Hospital, \\ Bangkok, Thailand
}

\begin{abstract}
Objective: The aim of this study was to demonstrate the efficacy of neoadjuvant androgen deprivation therapy (NADT) on perioperative outcomes in patients who underwent radical prostatectomy (RP).

Materials and Methods: From January 2008 to July 2018, we collected retrospective data of patients with clinically localized prostate cancer who underwent RP to assess their perioperative and pathological outcomes. The data included age, body mass index (BMI), serum prostate specific antigen (PSA) level, clinical stage, neoadjuvant ADT usage, operative time, estimated blood loss (EBL), perioperative complications, blood transfusion rate, adjacent organ injury rate, length of hospital stay, pathological stage, Gleason score (GS) of the biopsy and pathological specimen, specimen weight (g), and margin status.

Results: Of the 718 RPs performed, 138 (19.22\%) were NADT and 580 (80.78\%) were nonNADT. Patients who underwent NADT had a significant benefit in operative time (185 vs 195 mins), EBL (300 vs $500 \mathrm{~mL}$ ) and specimen weight. These benefits were more obvious in non-low risk prostate cancer with less operative time, EBL, blood transfusion rate, length of hospital stay and specimen weight. However, the margin status and adjacent organ injury rate were similar in the NADT and non-NADT groups.

Conclusion: NADT provides significantly better perioperative outcomes, especially in nonlow risk prostate cancer, and has comparable pathological outcomes.

Keywords: prostate cancer, neoadjuvant androgen deprivation therapy, radical prostatectomy, laparoscopy, robot-assisted laparoscopic surgery, open surgery, perioperative outcome
\end{abstract}

\section{Introduction}

Prostate cancer is the fifth most common cancer in Thai men, ${ }^{1}$ and the number of cases continues to increase despite active screening. Radical prostatectomy (RP) is the standard of care in the management of clinically localised cancer and also an option for the treatment of locally advanced prostate cancer. RP can be performed using open radical prostatectomy (ORP), laparoscopic radical prostatectomy (LRP), or robotic-assisted laparoscopic radical prostatectomy (RALRP) techniques.

Another recognised treatment for regional and metastatic prostate cancer is androgen deprivation therapy (ADT). ADT is also a beneficial supplement to external beam radiation therapy $(\mathrm{EBRT}){ }^{2}$ However, the use of neoadjuvant
Correspondence: Wisoot Kongchareonsombat

Division of Urology, Department of Surgery, Faculty of Medicine, Ramathibodi Hospital, Mahidol University, Bangkok, Thailand

Tel +66-2-20II536

Fax+66-89-8II 2436

Email wisoot2002@hotmail.com 
androgen deprivation therapy (NADT) prior to RP as a perioperative treatment is still controversial. ${ }^{2}$ Previous research has demonstrated that NADT lowers the positive margin status, but this benefit has not translated into improvements in long-term PSA-free survival. ${ }^{3}$ NADT is also associated with worrisome side effects, such as osteoporosis, hot flashes, sexual dysfunction, metabolic syndrome, gynecomastia, anaemia and cardiovascular mortality. For all these reasons, current guidelines recommend against the use of NADT prior to RP. ${ }^{2,4,5}$

In the past few years, a growing trend has emerged towards the utilization of NADT, as it can provide a pathological complete response, ${ }^{6}$ a potentially positive impact on recurrence rates, ${ }^{7}$ a significant decrease in prostate cancer-related death ${ }^{8}$ and fewer long-term effects on quality of life. ${ }^{9}$ The aim of the present study was to determine the benefit of NADT prior to RP in terms of perioperative outcomes.

\section{Materials and Methods}

\section{Population and Surgical Techniques}

Between January 2008 and July 2018, 718 prostate cancer patients were treated by RP at Ramathibodi Hospital in Thailand. Of these patients, 138 had undergone NADT (NADT group) and 580 had not undergone neoadjuvant ADT (non-NADT group). The remaining 2 patients were excluded from the study due to incomplete data. The principles of the Helsinki Declaration were followed during the study, consent and the confidentiality of the patients' data was maintained by the authority of the Committee for Research of the Faculty of Medicine, Ramathibodi Hospital, Mahidol University (date of approval: 20 February 2020, ID MURA2020/298).

The ORP was performed in a retropubic fashion via low midline incision (Figure 1). A retzius space was approached with blunt and sharp dissection along the outside of left umbilical ligament. Endopelvic fascia on both sides were bluntly opened, puboprostatic ligament was dissected and dorsal venous complex was sutured and ligated using Vicryl No.1. The bladder neck was incised with monopolar cautery then foley's catheter was pull and traction. The seminal vesicle and vas deferens were dissected. Denonvillier's fascia was opened and the posterior surface of prostate was freed. The lateral prostatic pedicles were dissected with monopolar cautery and ligated without nerve sparing, followed by incised of urethra by Metzenbaum scissor. An urethrovesical anastomosis was performed with interrupted sutures, using Vicryl 3/0 6 stitches. Before the last stitch was performed, new $20 \mathrm{Fr}$ Foley's catheter was inserted via urethra into bladder. Bleeding was checked and stopped. Silastic drain was placed in cul-de-sac.

The LRPs were performed in an extraperitoneal fashion using 5 trocars with some modification in port position to facilitate the prevention to graft injury (Figure 2). Subumbilical incision was done and extraperitoneal space was created using kidney-shaped balloons with (PDB, Covidien, United States). Retropneumoperitoneum was performed by $\mathrm{CO}_{2}$ insufflation to create at an abdominal pressure of $15 \mathrm{mmHg}$, followed by port placement as Figure 2, all trocars were inserted under direct visualization. Thirty-degree standard Trendelenburg position was used with cushioning for dependent zone. A Retzius space was developed. Endopelvic fascia on both sides were opened, followed by puboprostatic ligament was dissected and dorsal venous complex was controlled using Vicryl No.1 CT-1 needle. The bladder neck was incised with monopolar cautery then foley's catheter was pull and traction. The seminal vesicle and vas deferens were dissected. Denonvillier's fascia was opened and the posterior surface

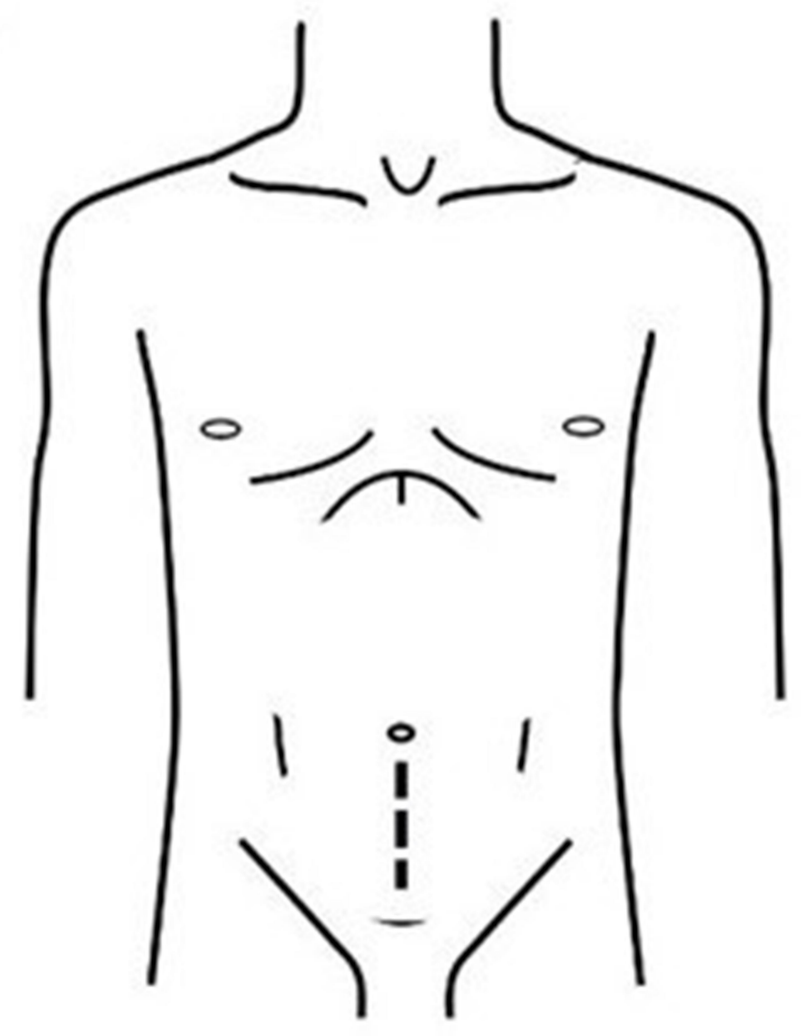

Figure I Incision of open radical prostatectomy. 


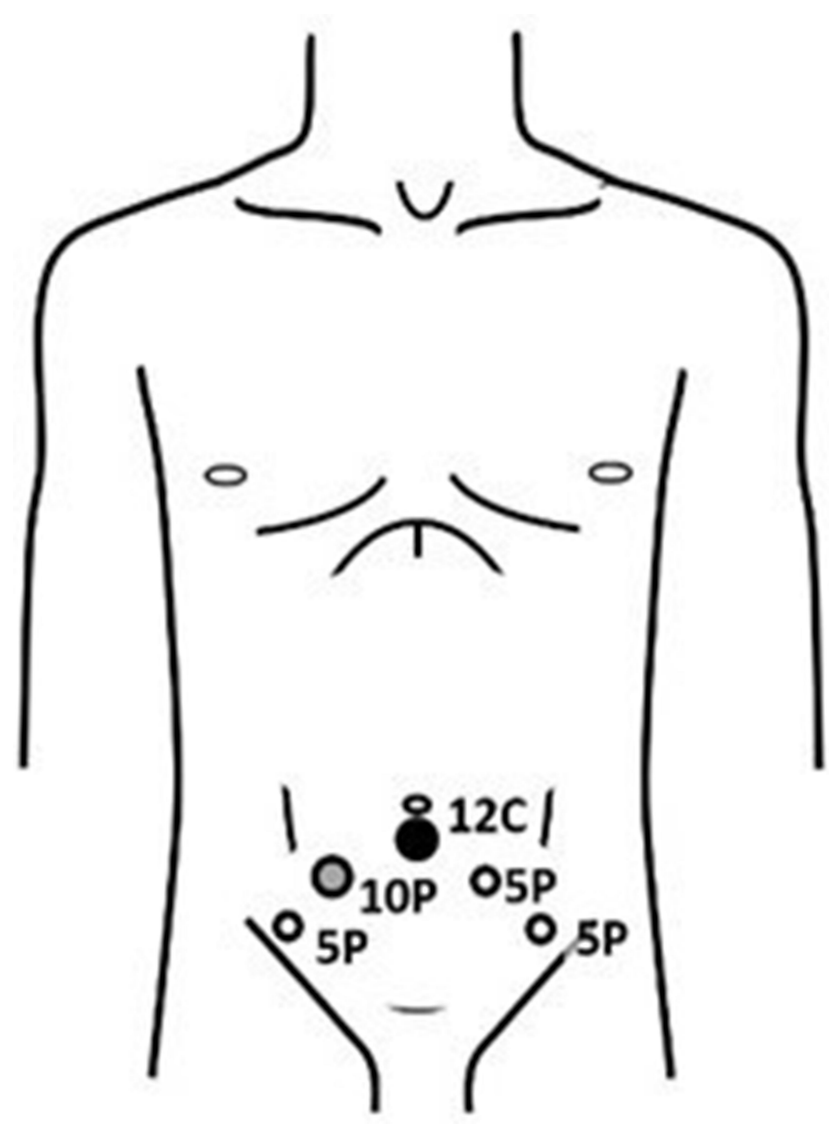

Figure 2 Port placement of laparoscopic radical prostatectomy.

of prostate was freed. The lateral prostatic pedicles were controlled by Hem-o-lock clip and dissected with vessel sealing device (LigaSure Impact Curved, Large jaw, Medtronic, United Kingdom) without nerve sparing, followed by incised of urethra by cold scissor. An urethrovesical anastomosis was performed with continuous watertight sutures, using Vicryl 3/0. Before passing the anterior stitch, the $20 \mathrm{Fr}$ Foley's catheter was passed into the bladder and the anastomosis was completed. A closed suction drain was placed in cul-de-sac. The specimen was retrieved with the use of laparoscopic bag through a subumbilical incision.

The RALRPs were performed with transperitoneal fashion using the da Vinci Si Surgical System, with 5 trocars. We place the trocars medially than the standard port site to deliver sufficient access without graft injury (Figure 3). The pneumoperitoneum pressure of $15 \mathrm{mmHg}$ was created after subumbilical puncture with Veress needle. A $12 \mathrm{~mm}$ subumbilical trocar was inserted into abdominal space and was used as a camera port. Two robotic trocars were placed on the right side with at least $8 \mathrm{~cm}$ apart from each other (Arm 1, Arm 3). On the left

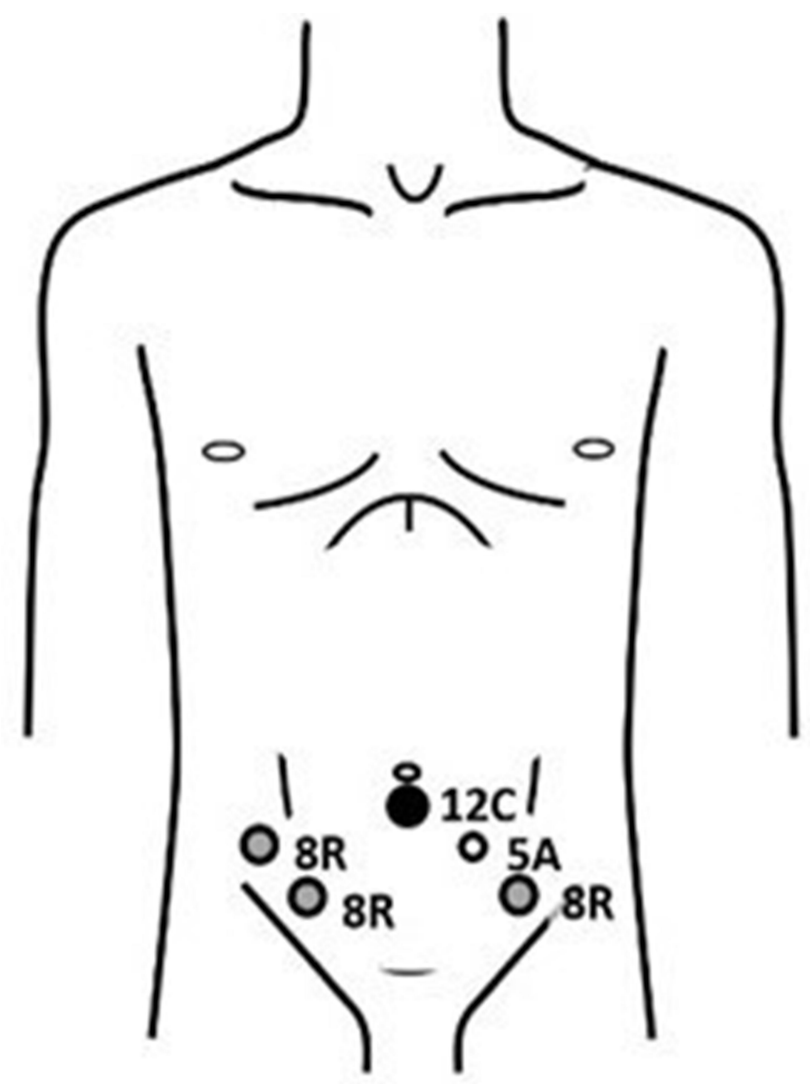

Figure 3 Port placement of robotic-assisted radical prostatectomy.

side, a robotic trocar was placed (Arm 2), and the assistance $12 \mathrm{~mm}$ trocar was inserted between the second port and camera port as Figure 3, all trocars were inserted under direct visualization. Each arm was equipped with Monopolar scissor (Arm 1), Bipolar Maryland (Arm 2) and Prograpse (Arm 3). Thirty-degree standard Trendelenburg position was used with cushioning for dependent zone. A Retzius space was developed. Endopelvic fascia on both sides were opened, followed by puboprostatic ligament was dissected and dorsal venous complex was controlled using barbed suture No.1 (V-Loc PBT wound closure device, Medtronic, United Kingdom). The bladder neck was incised with monopolar and bipolar cautery then foley's catheter was pull and traction. The seminal vesicle and vas deferens were dissected. Denonvillier's fascia was opened and the posterior surface of prostate was freed. The lateral prostatic pedicles were controlled by Hem-o-lock clip and dissected with monopolar and bipolar cautery with 1 case of interfacial nerve sparing and 2 case of non-nerve sparing, followed by incised of urethra by cold scissor. An urethrovesical anastomosis was performed with continuous watertight 
sutures, using barbed suture 3/0 (V-Loc PBT wound closure device, Medtronic, United Kingdom). Before passing the anterior stitch, the 20 Fr Foley's catheter was passed into the bladder and the anastomosis was completed. A closed suction drain was placed in cul-de-sac. The specimen was retrieved with the use of laparoscopic bag through a subumbilical incision.

Nerve-sparing RPs were performed in some cases, except when biopsy specimens revealed extensive cancer or in cases of preoperative poor-quality erections, current and future lack of a sexual relationship, or other medical conditions that could adversely affect erections (eg, hypertension, diabetes mellitus, neurologic diseases, psychiatric diseases or medications that produce erectile dysfunction). However, we shared the decision-making with the patients and the surgeon.

Six instructor surgeons participated in this study. Two surgeons always performed ORPs over 25 years, while the other four surgeons performed all three techniques as shown in Table 1. The procedure techniques were decided by shared decision-making with patients.

\section{Androgen Deprivation Therapy}

In this study, both gonadotropin-releasing hormone (GnRH) analogues, such as degarelix, and GnRH antagonists, such as leuprolide, goserelin and triptorelin, were used for the ADT. The choice of medication depended on the patient's insurance and the surgeon preference. The duration of NADT was 3 months, since some evidence indicates that this is the optimum duration. ${ }^{10,11}$

The decision was made upon the discussion between patients and physicians. Since the specific criteria for selection have not been officially stated, we typically

Table I Surgeons Participation

\begin{tabular}{|l|c|c|c|}
\hline Participant Surgeons: n (\%) & ORP & LRP & RALRP \\
\hline 2008 & 4 & 2 & 0 \\
2009 & 4 & 2 & 0 \\
2010 & 4 & 2 & 0 \\
2011 & 4 & 2 & 0 \\
2012 & 4 & 2 & 0 \\
2013 & 4 & 2 & 2 \\
2014 & 4 & 2 & 2 \\
2015 & 4 & 2 & 2 \\
2016 & 6 & 4 & 4 \\
2017 & 6 & 4 & 4 \\
2018 & 6 & 4 & 4 \\
\hline
\end{tabular}

Abbreviations: LRP, laparoscopic radical prostatectomy; ORP, open radical prostatectomy; RALRP, robotic-assisted radical prostatectomy. advise the NADT for those patients who a. associated with high or very high-risk disease and $b$. are on the waitlist for surgery longer than three months.

\section{Baseline Characteristics and Preoperative Parameters}

The following data were collected from all patients: age, body weight $(\mathrm{kg})$, height $(\mathrm{cm})$, body mass index (BMI), prostate-specific antigen (PSA) level, underlying disease, clinical stage (TNM classification), prostate cancer risk group (National Comprehensive Cancer Network [NCCN] classification), ${ }^{2}$ and the Gleason score (GS) of the biopsy specimen.

\section{Perioperative Outcomes}

Perioperative outcomes included operative time (minutes); estimated blood loss (EBL) (mL); perioperative complications, including transfusion rate; adjacent organ injury of the bladder, rectum, ureter, bowel, or blood vessel; and length of hospital stay (days) (determined by subtracting the date of admission from the date of discharge).

\section{Pathological Outcomes}

All specimens were evaluated in accordance with the NCCN guidelines by an experienced uropathologist, who reported the prostate weight $(\mathrm{g})$, pathological stage, GS of the specimen and the margin status. A positive surgical margin (PSM) was defined as cancer cells extending to the inked surface of the specimen. ${ }^{12}$

\section{Statistical Analysis}

A descriptive study was performed. The data were analysed using a Wilcoxon rank-sum (Mann-Whitney) and Fisher's exact test to identify the statistical significance of the difference in means \pm standard deviation, median (interquartile range), and proportions. Analysis was performed using Stata version 14 software, with a $p$-value of $<0.005$ considered to be statistically significant.

\section{Results}

The demographic data and preoperative parameters are presented in Table 2. The non-NADT and NADT groups were not statistically different in terms of median age (68 vs 68 years; $p=0.819$ ), median body weight (67.1 vs 67.1 $\mathrm{kg} ; p=0.807)$, median height (165 vs $165 \mathrm{~cm} ; p=0.403$ ) or median BMI (24.4 vs $\left.24.3 \mathrm{~kg} / \mathrm{m}^{2} ; p=0.218\right)$. The pre- 
Table 2 Demographic Data and Pre-Operative Parameters of Non-NADT and NADT

\begin{tabular}{|c|c|c|c|}
\hline Demographic Data & $\begin{array}{c}\text { Non-NADT } \\
(n=580)\end{array}$ & $\begin{array}{c}\text { NADT } \\
(n=138)\end{array}$ & p-value \\
\hline \multicolumn{4}{|l|}{ Approach } \\
\hline ORP, n (\%) & $109(18.8)$ & $19(13.8)$ & $0.002^{\gamma}$ \\
\hline LRP, n (\%) & $208(35.9)$ & $33(23.9)$ & \\
\hline RALRP, n (\%) & $263(45.3)$ & $86(62.3)$ & \\
\hline Age (yrs.): median (range) & $68(63-72)$ & $68(63-72)$ & 0.819 \\
\hline Body weight (kgs.): median (range) & $67.1(61.6-73.4)$ & 67 (6I.I-74.5) & 0.807 \\
\hline Height (cms.): median (range) & $165(161-170)$ & $165(162-168)$ & 0.403 \\
\hline BMI (kg/m²): median (range) & $24.4(22.5-26.6)$ & $24.3(22.3-27.5)$ & 0.651 \\
\hline PSA Pre-op (ng/mL): median (range) & $10.1(7.2-15.2)$ & $33.4(16.2-56.6)$ & $<0.00 I^{*}$ \\
\hline \multicolumn{4}{|l|}{ Underlying: $n(\%)$} \\
\hline Hypertension & $350(60.5)$ & $85(61.6)$ & 0.847 \\
\hline Diabetes Mellitus & $129(22.3)$ & $4 \mathrm{I}(29.7)$ & 0.075 \\
\hline Dyslipidemia & $213(36.8)$ & $53(38.4)$ & 0.769 \\
\hline Other & $203(35.0)$ & $50(36.2)$ & 0.843 \\
\hline \multicolumn{4}{|l|}{ Clinical stage: n (\%) } \\
\hline Tla & $5(0.9)$ & $0(0)$ & $<0.001^{\gamma}$ \\
\hline TIb & $3(0.5)$ & $0(0)$ & \\
\hline TIc & $522(91.4)$ & $88(64.7)$ & \\
\hline $\mathrm{T} 2 \mathrm{a}$ & - & - & \\
\hline T3a & $20(3.5)$ & $14(10.3)$ & \\
\hline T3b & $21(3.7)$ & $33(24.3)$ & \\
\hline T3c & - & - & \\
\hline T4 & $0(0)$ & I (0.7) & \\
\hline GS from biopsy: median (range) & $7(6-7)$ & $8(7-9)$ & $<0.001 *$ \\
\hline $\mathbf{G S} \leq \mathbf{6}: \mathrm{n}(\%)$ & $238(4 I .0)$ & $21(15.2)$ & \\
\hline GS $=3+4: n(\%)$ & $142(24.5)$ & $14(10.1)$ & \\
\hline GS = 4+3: $n(\%)$ & $98(16.9)$ & $21(15.2)$ & \\
\hline GS = 8: n (\%) & $60(10.3)$ & $26(18.8)$ & \\
\hline GS = 9, I0: n (\%) & $24(4.1)$ & $45(32.6)$ & \\
\hline
\end{tabular}

Notes: *Comparison of groups by Wilcoxon rank-sum (Mann-Whitney) test; Data are presented as median (range); ${ }^{\gamma}$ Comparison of groups by Fisher's exact test; Data are presented as number (percent, \%).

Abbreviations: BMI, body mass index; GS, Gleason score; LRP, laparoscopic radical prostatectomy; NADT, neoadjuvant androgen deprivation therapy; ORP, open radical prostatectomy; PSA, prostatic-specific antigen; RALRP, robotic-assisted radical prostatectomy.

operative PSA level and GS of the biopsy specimens were statistically significantly higher in the NADT than in the non-NADT group (33.4 [16.2-56.6] vs 10.1 [7.2-15.2]; $p$ $=<0.001$ and 8 [7-9] vs $7[6-7] ; p=<0.001)$. The clinical stage differed significantly between the two groups $(p=$ $<0.001)$.

The perioperative outcomes (Table 3) showed that the operative time was significantly lower in the NADT than in the non-NADT group (185 vs 195 minutes; $p=<0.018$ ). The EBL was also significantly lower in the NADT than in the non-NADT group ( $300 \mathrm{vs} 500 \mathrm{~mL} ; p=<0.001$ ), but no difference was noted in the blood transfusion rate between the two groups. No statistically significant differences were detected between the NADT and non-NADT groups for adjacent organ injury rate $(2.9 \%$ vs $2.2 \% ; p=0.999)$ or length of hospital stay ( 6 vs 6 days; $p=0.184$ ). The most common site of injury was the rectum (11 patients).

The pathological outcomes (Table 4) revealed significant differences in pathological stage between the two groups $(p=0.001)$. The GS of the specimens was significantly higher in the NADT group than in the non-NADT (7 [7-9] vs 7 [7-7]; $p<0.001$ ). The specimen weight was significantly lower in the NADT than in the non-NADT group (34.8 vs $39.5 ; p=0.014$ ). The PSM did not differ 
Table 3 Perioperative Outcomes of Non-NADT and NADT

\begin{tabular}{|c|c|c|c|}
\hline Perioperative Outcomes & $\begin{array}{c}\text { Non-NADT } \\
(n=580)\end{array}$ & $\begin{array}{c}\text { NADT } \\
(n=138)\end{array}$ & p-value \\
\hline Operative time (mins): median (range) & $195(155-255)$ & $185(150-225)$ & $0.018^{*}$ \\
\hline Estimate blood loss ( $\mathrm{mL}$ ): median (range) & $500(300-1000)$ & $300(200-600)$ & $<0.001 *$ \\
\hline $\begin{array}{l}\text { Blood transfusion: } \\
\text { n (\%) }\end{array}$ & $136(24.2)$ & $24(17.4)$ & 0.091 \\
\hline $\begin{array}{l}\text { Adjacent organ injury: } \\
\mathrm{n}(\%)\end{array}$ & $16(2.9)$ & $3(2.2)$ & 0.999 \\
\hline \multicolumn{4}{|l|}{ Adjacent organ injury: $n(\%)$} \\
\hline Bladder & 3 & - & - \\
\hline Rectum & 11 & - & - \\
\hline Left ureter & - & 1 & - \\
\hline Right ureter & - & 2 & - \\
\hline Right external iliac vein & I & - & - \\
\hline Small bowel & I & - & - \\
\hline Hospitalization time (day): median (IQR) & $6(5-9)$ & $6(5-8)$ & 0.184 \\
\hline
\end{tabular}

Notes: *Comparison of groups by Wilcoxon rank-sum (Mann-Whitney) test; Data are presented as median (range).

Abbreviations: IQR, interquartile range; NADT, neoadjuvant androgen deprivation therapy.

Table 4 Pathological Outcomes of Non-NADT and NADT

\begin{tabular}{|c|c|c|c|}
\hline Clinical Outcomes & $\begin{array}{c}\text { Non-NADT } \\
(n=580)\end{array}$ & $\begin{array}{c}\text { NADT } \\
(n=138)\end{array}$ & p-value \\
\hline \multicolumn{4}{|l|}{ Pathological stage: n (\%) } \\
\hline $\mathrm{T} 2 \mathrm{a}$ & $67(11.6)$ & $2(1.5)$ & $<0.00 I^{\gamma}$ \\
\hline $\mathrm{T} 2 \mathrm{~b}$ & $22(3.8)$ & $\mathrm{I}(0.7)$ & \\
\hline $\mathrm{T} 2 \mathrm{c}$ & $263(45.7)$ & $49(35.8)$ & \\
\hline T3a & $130(22.6)$ & $28(20.4)$ & \\
\hline T3b & $93(16.2)$ & $57(4 \mid .6)$ & \\
\hline T4 & I (0.2) & $0(0)$ & \\
\hline Pathologic GS: median (range) & $7(7-7)$ & $7(7-9)$ & $<0.00 I^{*}$ \\
\hline $\mathbf{G S} \leq \mathbf{6 :} \mathrm{n}(\%)$ & $132(22.8)$ & $9(6.5)$ & \\
\hline GS = 3+4: $n(\%)$ & $204(35.2)$ & $34(24.6)$ & \\
\hline GS $=4+3: n(\%)$ & $124(2 \mid .4)$ & $25(18.1)$ & \\
\hline GS = 8: n (\%) & $5 \mathrm{I}(8.8)$ & $22(15.9)$ & \\
\hline GS = 9, 10: n (\%) & $65(11.2)$ & $46(33.3)$ & \\
\hline Prostate volume (g): median (IQR) & $39.5(30-50)$ & $34.8(28.5-44.2)$ & $0.014 *$ \\
\hline Positive Margin: n (\%) & $198(36.4)$ & $57(43.9)$ & 0.131 \\
\hline
\end{tabular}

Notes: *Comparison of groups by Wilcoxon rank-sum (Mann-Whitney) test; Data are presented as median (range); ${ }^{\gamma}$ Comparison of groups by Fisher's exact test; Data are presented as number (percent, \%).

Abbreviations: GS, Gleason score; IQR, interquartile range; NADT, neoadjuvant androgen deprivation therapy. 
significantly between the NADT and non-NADT group $(36.4 \%$ vs $43.9 \% ; p=0.131)$.

Subgroup analysis for perioperative and pathological outcomes of the non-low risk prostate cancer patients are presented in Table 5. The perioperative outcomes, which included operative time, EBL, blood transfusion rate and length of hospital stay, were significantly better in the NADT than in the non-NADT group. Only adjacent organ injury rate showed no significant difference in the two groups. The pathological stage was significantly different in the two groups $(p<0.001)$. The GS of the specimens was significantly higher in the NADT group (7.5 [7-9] vs 7 [7-8]; $p<0.001$ ) and the specimen weight was significantly lower in the NADT group $(35$ [29.1-44] $\mathrm{mL}$ vs 40 [32.2-51.5] mL; $p=0.002$ ). However, the PSM still did not show a significant difference between the NADT and non-NADT groups (43.6\% vs $47.8 \% ; p=0.506)$.

\section{Discussion}

Neoadjuvant therapy is the current standard of care for several solid tumour malignancies, including bladder, breast, and rectal cancer. NADT prior to surgery may downstage a malignancy to facilitate surgical resection, thereby lowering the positive surgical margin rate and improving perioperative outcomes. ${ }^{13,14}$ In our study, the median operative time was significantly lower in the NADT than in the non-NADT group $(\mathrm{p}=0.018)$ and a lower EBL was also observed in the NADT group $(\mathrm{p}<0.001)$.

Androgen deprivation therapy affects tumour behaviour and biology by changing the metabolic patterns of atrophy, lowering serum PSA level and reducing histopathologic atrophy. ${ }^{3}$ These tumour responses may have contributed to the smaller prostate volume in the NADT group than in the non-NADT group (39.5 vs $34.8 \mathrm{~g}, p=$ 0.014 ), since enlarged prostate glands usually have more vascularity and a wider resection margin. ${ }^{15-17}$

The transfusion rate did not differ significantly between the two groups $(p=0.091)$. This may reflect the practice in our hospital of having the anaesthesiologist initiate transfusion when the haematocrit drops below 30 . Consequently, some patients with a higher baseline haematocrit would be less likely to receive a transfusion.

Table 5 Perioperative Outcomes and Pathological Outcomes of PCa Non-Low Risk Group

\begin{tabular}{|c|c|c|c|}
\hline Perioperative and Pathological Outcomes & $\begin{array}{c}\text { Non-NADT } \\
(n=308)\end{array}$ & $\begin{array}{l}\text { NADT } \\
(n=122)\end{array}$ & p-value \\
\hline Operative time (mins): median (range) & $205(165-257.5)$ & $185(150-225)$ & $0.006 *$ \\
\hline Estimate blood loss (mL): median (range) & $500(300-1000)$ & $300(200-600)$ & $<0.00 I^{*}$ \\
\hline $\begin{array}{l}\text { Blood transfusion: } \\
\text { n (\%) }\end{array}$ & $81(26.6)$ & $21(17.2)$ & $0.044^{*}$ \\
\hline $\begin{array}{l}\text { Adjacent organ injury: } \\
\mathrm{n}(\%)\end{array}$ & $10(3.3)$ & $3(2.5)$ & 0.764 \\
\hline Hospitalization time (day): median (IQR) & $6(5-9)$ & $6(5-8)$ & $0.036^{*}$ \\
\hline \multicolumn{4}{|l|}{$\begin{array}{l}\text { Pathological stage: } \\
\text { n (\%) }\end{array}$} \\
\hline $\mathrm{T} 2 \mathrm{a}$ & $22(7.2)$ & $\mathrm{I}(0.8)$ & $<0.001^{\gamma}$ \\
\hline $\mathrm{T} 2 \mathrm{~b}$ & $9(2.9)$ & $\mathrm{I}(0.8)$ & \\
\hline $\mathrm{T} 2 \mathrm{c}$ & $124(40.5)$ & $4 I(33.6)$ & \\
\hline T3a & $78(25.5)$ & $25(20.5)$ & \\
\hline $\mathrm{T} 3 \mathrm{~b}$ & $72(23.5)$ & $54(44.3)$ & \\
\hline $\mathrm{T} 4$ & I $(0.3)$ & $0(0)$ & \\
\hline Pathologic GS: median (range) & $7(7-8)$ & 7.5 (7-9) & $<0.00 I^{*}$ \\
\hline Prostate volume (g): median (IQR) & $40(32.2-51.5)$ & $35(29.1-44)$ & $0.002 *$ \\
\hline Positive Margin: n (\%) & $126(43.6)$ & $55(47.8)$ & 0.506 \\
\hline
\end{tabular}

Notes: *Comparison of groups by Wilcoxon rank-sum (Mann-Whitney) test; Data are presented as median (range); ${ }^{\gamma}$ Comparison of groups by Fisher's exact test; Data are presented as number (percent, \%).

Abbreviations: IQR, interquartile range; GS, Gleason score; NADT, neoadjuvant androgen deprivation therapy. 
The length of hospital stay also did not differ statistically between the two groups. However, the hospital stays were longer in the present study than in a previous study by Wallerstedt et al, ${ }^{18}$ who reported lengths of hospital stay of around 3.3-4.1 days. This discrepancy can be attributed to an institutional practice where patients are routinely discharged after the closed-suction pelvic drain is removed. However, many other factors, such as socioeconomic status, inexpensive room rates, anxiety and patient pain tolerance, might affect the length of a hospital stay.

No pathological down-staging could be demonstrated in the present study because of the limited pre-operative $\mathrm{T}$ clinical staging. In our setting, pre-operative imaging is not generally affordable for all patients, so many patients are classified as T1c (91.4\%). Nevertheless, the data show a decrease in the median GS in the NADT group from the pre-operative biopsy specimen score of $8^{7-9}$ to the postoperative specimen score of $7 .^{7-9}$

The oncological control of RP in prostate cancer can be measured by the PSM, biochemical recurrence (BCR) rate, time to biochemical recurrence, local recurrence and distant metastasis. ${ }^{19}$ Sachdeva et $\mathrm{al}^{20}$ and other researchers ${ }^{21-24}$ have shown that a PSM in prostate cancer is considered an adverse oncologic outcome and is associated with an increased likelihood of BCR. However, the significant predictors of BCR are tumour volume, a high GS and a high pre-operative PSA level. In our study series, no statistically significant difference was noted in PSM between both groups, which is consistent with the results of many guidelines and the current literature. ${ }^{2,45}$ The PSM rate in large series data ranged from $11 \%$ to $50 \%{ }^{11}$ and from $12.1 \%$ to $41.3 \%$ in a recent meta-analysis. ${ }^{12}$ The PSM rate in our study was higher, which may reflect the participation of multiple surgeons in this study, as each surgeon may have had a different learning curve. For example, in the present study, two new instructor surgeons had just started performing RALRP in 2016. In addition, after the subgroup analysis, most of the PSMs were in the T3 stage, indicating that PSMs could result from the nature of the cancer that had extended beyond the prostatic capsule.

The subgroup analysis revealed that the perioperative and pathological outcomes significantly better almost all parameters in the non-low risk NADT group. This can be related to the fact that study was heterogenous and not weighted towards those who may benefit the most from NADT.

Patients with PSM are typically given two treatment options: external beam radiation therapy (proton beam radiation) with or without androgen deprivation therapy (ADT) or observation. Unfortunately, data related to the rate of conversion and catheterisation time could not be collected to determine our patient outcomes, as this study was retrospective. The functional outcomes, such as incontinence and erectile dysfunction, are presently being assembled by the authors, who will report on these findings in a subsequent study.

Author want to underline some interesting upcoming trend from the Pignot and Walz, ${ }^{25}$ and we comply with their findings that the multimodal approach including both local and systemic treatment neoadjuvant may be an option for selected patients especially with non-low risk prostate cancer. However, there is still not enough data to conclude when is the best setting for treatment (Neoadjuvant/adjuvant) and who would really benefit from treatment.

The present study has some limitations. One was its retrospective nature, which compared the effect of NADT with non-NADT but did not include data regarding the type of $\mathrm{ADT}$, since no standard regimens for NADT currently exist. Consequently, the choice of treatment for each patient is highly heterogeneous and depends on patient insurance and physician preference. A second limitation is that all RPs were performed by different surgeons, raising the possibility of bias in the process of evaluating the procedure outcomes. A third limitation is that this study lacked data about the oncological and functional follow-ups. A fourth limitation is that this study had some significant differences in baseline characteristic, approach, PSA pre-op and GS from biopsy (33.4 [16.2-56.6] vs 10.1 [7.2-15.2]; $p=$ $<0.001$ and 8 [7-9] vs 7 [6-7]; $p=<0.001$ ), as a result of the selection bias by physician typically offer treatment with NADT in high or very high-risk disease and the trend toward the Robotic-assisted era which can confound the effect of NADT. The findings of the present study would be strengthened by conducting a prospective randomised study with a higher case volume, as this would reduce biases and provide much more accurate results. 


\section{Conclusion}

This study has demonstrated the long-term data on the use of NADT prior to RP and has shown an association between NADT and a positive effect on perioperative outcomes in the prostate cancer non-low risk group. Further randomized, prospective studies are needed to elucidate the true effect of NADT on perioperative outcomes, pathological outcomes and survival benefit.

\section{Acknowledgments}

This work was supported by Wijittra Matang, Yada Phengsalae and Kornkanok Somboonpun. The authors thank Wijittra Matang, Yada Phengsalae and Kornkanok Somboonpun for continued support and encouragement.

\section{Disclosure}

The authors declare no conflicts of interest in this work.

\section{References}

1. National cancer institute Doms, Thailand. Hospital base cancer registry 2015. 2017.

2. network NCC. Prostate cancer (Version 1.2020). Available from: https://www.nccn.org/professionals/physician_gls/pdf/prostate.pdf. Accessed May 14, 2021.

3. Meng MV. Treatment of Locally Advance Prostate Cancer. 12th ed. Partin AW, editor. Elsevier; 2020:2.

4. Sanda MG, Chen RC, Crispino T, et al. Clinically localized prostate cancer: AUA/ASTRO/SUO guideline; 2017. Available from: https:// www.auanet.org/guidelines/prostate-cancer-clinically-localized-guide line\#x14226. Accessed May 14, 2021

5. Mottet N, Bellmunt J, Briers E, et al. EAU - ESTRO - ESUR SIOG guidelines on prostate cancer; 2020. Available from: https:// uroweb.org/guideline/prostate-cancer/. Accessed May 14, 2021.

6. Montgomery B, Tretiakova MS, Joshua AM, et al. Neoadjuvant enzalutamide prior to prostatectomy. Clin Cancer Res. 2017;23 (9):2169-2176. doi:10.1158/1078-0432.CCR-16-1357

7. McKay RR, Montgomery B, Xie W, et al. Post prostatectomy outcomes of patients with high-risk prostate cancer treated with neoadjuvant androgen blockade. Prostate Cancer Prostatic Dis. 2018;21 (3):364-372. doi:10.1038/s41391-017-0009-6

8. Tosco L, Laenen A, Briganti A, et al. The survival impact of neoadjuvant hormonal therapy before radical prostatectomy for treatment of high-risk prostate cancer. Prostate Cancer Prostatic Dis. 2017;20 (4):407-412. doi:10.1038/pcan.2017.29

9. Chen MC, Kilday PS, Elliott PA, et al. Neoadjuvant leuprolide therapy with radical prostatectomy: long-term effects on healthrelated quality of life. Eur Urol Focus. 2020. doi:10.1016/j. euf.2020.03.001

10. Meyer F, Bairati I, Bedard C, Lacombe L, Tetu B, Fradet Y. Duration of neoadjuvant androgen deprivation therapy before radical prostatectomy and disease-free survival in men with prostate cancer. Urology. 2001;58(2 Suppl 1):71-77. doi:10.1016/S0090-4295(01) 01245-6
11. Soloway MS, Pareek K, Sharifi R, et al. Neoadjuvant androgen ablation before radical prostatectomy in $\mathrm{cT} 2 \mathrm{bNxMo}$ prostate cancer: 5-year results. J Urol. 2002;167(1):112-116. doi:10.1016/S00225347(05)65393-1

12. Silberstein JL, Eastham JA. Significance and management of positive surgical margins at the time of radical prostatectomy. Indian J Urol. 2014;30(4):423-428. doi:10.4103/0970-1591.134240

13. Gómez Veiga F, Lorenzo Patiño MJ, Díaz Bermúdez J, et al. [Effect of complete androgen block before radical prostatectomy for cancer of the prostate]. Arch Esp Urol. 1997;50(4):355-363.

14. Polito M, Muzzonigro G, Minardi D, Montironi R. Effects of neoadjuvant androgen deprivation therapy on prostatic cancer. Eur Urol. 1996;30(Suppl 1):26-31. doi:10.1159/000474242

15. Frota R, Turna B, Santos BM, Lin YC, Gill IS, Aron M. The effect of prostate weight on the outcomes of laparoscopic radical prostatectomy. BJU Int. 2008;101(5):589-593. doi:10.1111/j.1464410X.2007.07263.X

16. Hsu EI, Hong EK, Lepor H. Influence of body weight and prostate volume on intraoperative, perioperative, and postoperative outcomes after radical retropubic prostatectomy. Urology. 2003;61(3):601-606. doi:10.1016/S0090-4295(02)02422-6

17. Kim MS, Jang WS, Chung DY, et al. Effect of prostate gland weight on the surgical and oncological outcomes of extraperitoneal robotassisted radical prostatectomy. BMC Urol. 2019;19(1):1. doi:10.1186/ s12894-018-0434-4

18. Wallerstedt A, Tyritzis SI, Thorsteinsdottir T, et al. Short-term results after robot-assisted laparoscopic radical prostatectomy compared to open radical prostatectomy. Eur Urol. 2015;67(4):660-670. doi:10.1016/j.eururo.2014.09.036

19. Akand M, Celik O, Avci E, Duman I, Erdogru T. Open, laparoscopic and robot-assisted laparoscopic radical prostatectomy: comparative analysis of operative and pathologic outcomes for three techniques with a single surgeon's experience. Eur Rev Med Pharmacol Sci. 2015;19(4):525-531.

20. Sachdeva A, Veeratterapillay R, Voysey A, et al. Positive surgical margins and biochemical recurrence following minimally-invasive radical prostatectomy - an analysis of outcomes from a UK tertiary referral centre. BMC Urol. 2017;17. doi:10.1186/s12894-017-0262-y

21. De La Roca RL, Da Cunha IW, Bezerra SM, Da Fonseca FP. Radical prostatectomy and positive surgical margins: relationship with prostate cancer outcome. Int Braz J Urol. 2014;40(3):306-315. doi:10.1590/S1677-5538.IBJU.2014.03.03

22. Meeks JJ, Eastham JA. Radical prostatectomy: positive surgical margins matter. Urol Oncol. 2013;31(7):974-979. doi:10.1016/j. urolonc.2011.12.011

23. Pettenati C, Neuzillet Y, Radulescu C, Herve JM, Molinie V, Lebret T. Positive surgical margins after radical prostatectomy: what should we care about? World J Urol. 2015;33(12):1973-1978. doi:10.1007/ s00345-015-1580-x

24. Yossepowitch O, Briganti A, Eastham JA, et al. Positive surgical margins after radical prostatectomy: a systematic review and contemporary update. Eur Urol. 2014;65(2):303-313. doi:10.1016/j. eururo.2013.07.039

25. Pignot G, Walz J. Identifying the relevant population for neoadjuvant chemo-hormonal therapy combined with radical prostatectomy. Gland Surg. 2020;9(2):495-497. doi:10.21037/gs.2019.12.22 


\section{Publish your work in this journal}

Research and Reports in Urology is an international, peer-reviewed, open access journal publishing original research, reports, editorials, reviews and commentaries on all aspects of adult and pediatric urology in the clinic and laboratory including the following topics: Pathology, pathophysiology of urological disease; Investigation and

Submit your manuscript here: https://www.dovepress.com/research-and-reports-in-urology-journ treatment of urological disease; Pharmacology of drugs used for the treatment of urological disease. The manuscript management system is completely online and includes a very quick and fair peer-review system, which is all easy to use. Visit http://www.dovepress.com/ testimonials.php to read real quotes from published authors. 